\title{
Borel equivalence relations and cardinal algebras
}

\author{
Alexander S. Kechris and Henry L. Macdonald
}

\section{Introduction}

(A) In the late 1940's Tarski published the book Cardinal Algebras, see [T], in which he developed an algebraic approach to the theory of cardinal addition, devoid of the use of the full Axiom of Choice, which of course trivializes it. A cardinal algebra is an algebraic system consisting of an abelian semigroup with identity (viewed additively) augmented with an infinitary addition operation for infinite sequences, satisfying certain axioms.

The theory of cardinal algebras seems to have been largely forgotten but our goal in this paper is to show that they appear naturally in the context of the current theory of Borel equivalence relations, as can be verified by rather elementary considerations. As a result one can apply Tarski's theory to discover a number of interesting laws governing the structure of Borel equivalence relations, which, in retrospect rather surprisingly, have not been realized before.

Below if $E, F$ are Borel equivalence relations on standard Borel spaces $X, Y$, resp., a Borel reduction of $E$ to $F$ is a Borel function $f: X \rightarrow Y$ such that

$$
x E y \Longleftrightarrow f(x) F f(y) .
$$

Then $f$ induces an injection $[f]: X / E \rightarrow Y / F$, defined by $[f]\left([x]_{E}\right)=[f(x)]_{F}$. We denote by

$$
E \leq_{B} F
$$

the pre-order of Borel reducibility, defined by

$$
E \leq_{B} F \Longleftrightarrow \text { there is a Borel reduction of } E \text { to } F \text {. }
$$


We also let

$$
E<_{B} F \Longleftrightarrow E \leq_{B} F \& F \not_{B} E
$$

and

$$
E \sim_{B} F \Longleftrightarrow E \leq_{B} F \& F \leq_{B} E
$$

for the associated notion of Borel bi-reducibility. Finally we let

$$
E \cong{ }_{B} F
$$

denote Borel isomorphism.

If $n>0$ is a positive integer and $E$ a Borel equivalence relation, then $n E$ is the direct sum of $n$ copies of $E$, i.e., the equivalence relation $F$ on $X \times\{0,1, \ldots, n-1\}$ (where $E$ lives on $X$ ), defined by $(x, i) F(y, j) \Longleftrightarrow$ $x E y \& i=j$.

Recall also that a Borel equivalence relation $E$ is countable if every E-class is countable.

In order to give the flavor of the results one can obtain by applying Tarski's theory to cardinal algebras associated with Borel equivalence relations, we mention a few representative examples of results that will be discussed later (in much more general forms, see Theorem 2.2 and Section 3, (B)).

Theorem 1.1. (i) (Existence of least upper bounds) Any increasing sequence $F_{0} \leq_{B} F_{1} \leq_{B} \ldots$ of countable Borel equivalence relations has a least upper bound (in the pre-order $\leq_{B}$ ).

(ii) (Interpolation) If $\mathcal{S}, \mathcal{T}$ are countable sets of countable Borel equivalence relations and $\forall E \in \mathcal{S} \forall F \in \mathcal{T}\left(E \leq_{B} F\right)$, then there is a countable Borel equivalence relation $G$ such that $\forall E \in \mathcal{S} \forall F \in \mathcal{T}\left(E \leq_{B} G \leq_{B} F\right)$.

(iii) (Cancellation) If $n>0$ and $E, F$ are countable Borel equivalence relations, then

$$
n E \leq_{B} n F \Longrightarrow E \leq_{B} F
$$

and therefore

$$
n E \sim_{B} n F \Longrightarrow E \sim_{B} F .
$$

Also if E, $F$ are arbitrary Borel equivalence relations, then

$$
n E \cong_{B} n F \Longrightarrow E \cong_{B} F
$$

(iv) (Dichotomy for integer multiples) For any countable Borel equivalence relation $E$, exactly one of the following holds: 
(a) $E<_{B} 2 E<_{B} 3 E<_{B} \ldots$,

(b) $\forall m>0 \forall n>0\left(m E \sim_{B} n E\right)$.

(B) This paper is organized as follows. In Section 2, we review the theory of cardinal algebras. In Section 3, we discuss various cardinal algebras that arise in the theory of Borel equivalence relations and, in combination with the results mentioned in Section 2, we derive various consequences about the structure of certain classes of Borel equivalence relations. In Section 4, we show, using ergodic theory, that the multiplicative analog of the additive cancellation law in Theorem 1.1 fails for countable Borel equivalence relations.

\section{Cardinal Algebras}

(A) A cardinal algebra, see [T], is a system $\left\langle A,+, \sum\right\rangle$, where $\langle A,+\rangle$ is an abelian semigroup with identity, which will be denoted by 0 , and $\sum: A^{\mathbb{N}} \rightarrow$ $A$ is an infinitary operation, satisfying the following axioms, where we put $\sum_{n<\infty} a_{n}=\sum\left(\left(a_{n}\right)_{n \in \mathbb{N}}\right)$ :

(A) $\sum_{n<\infty} a_{n}=a_{0}+\sum_{n<\infty} a_{n+1}$.

(B) $\sum_{n<\infty}\left(a_{n}+b_{n}\right)=\sum_{n<\infty} a_{n}+\sum_{n<\infty} b_{n}$.

(C) If $a+b=\sum_{n<\infty} c_{n}$, then there are $\left(a_{n}\right),\left(b_{n}\right)$ such that

$$
a=\sum_{n<\infty} a_{n}, b=\sum_{n<\infty} b_{n}, c_{n}=a_{n}+b_{n} .
$$

(D) If $\left(a_{n}\right),\left(b_{n}\right)$ are such that $a_{n}=b_{n}+a_{n+1}$, then there is $c$ such that for each $n, a_{n}=c+\sum_{i<\infty} b_{n+i}$.

Remark 2.1. These axioms are slightly different than the ones in [T, Definition 1.1] but they are equivalent.

For any natural number $n$ and finite sequence $\left(a_{i}\right)_{i<n}$ one can define $\sum_{i<n} a_{i}$ either by induction on $n$, using the addition operation + , or as $\sum_{i<\infty} b_{i}$, where $b_{i}=a_{i}$ for $i<n$ and $b_{i}=0$ for $i \geq n$, and these turn out to be the same. By convention when $n=0$ this sum is equal to 0 . 
For a natural number $n$ and any $a$, we define

$$
n a=\sum_{i<n} a,
$$

so that is particular $0 a=0$. Also we let

$$
\infty a=\sum_{n<\infty} a,
$$

Let also

$$
a \leq b \Longleftrightarrow \exists c(a+c=b) .
$$

It turns out that this is a partial ordering. Moreover all the expected commutativity, associativity laws for,$+ \sum$ and monotonicity with respect to $\leq$ hold (see [T, Section 1]).

Finally, for any finite or infinite family $\left(a_{i}\right)_{i<n}$, where $n \leq \infty$, we let $\bigwedge_{i<n} a_{i}$ be the infimum of this family in the poset $\langle A, \leq\rangle$, if it exists, and we define similarly the supremum $\bigvee_{i<n} a_{i}$.

(B) In [T, Sections 2-4] Tarski derives various laws that hold in any cardinal algebra. We list below those laws that appear most interesting in the application to Borel equivalence relations in Section 3.

Theorem 2.2 (Tarski). The following hold in any cardinal algebra $\left\langle A,+, \sum\right\rangle$ :

1. [ $\mathrm{T}, 2.24]$ If $a_{0} \leq a_{1} \leq a_{2} \ldots$, then $\bigvee_{n<\infty} a_{n}$ exists.

2. $[\mathrm{T}, 2.21,3.19] \bigvee_{n<\infty} \sum_{i<n} a_{i}=\sum_{i<\infty} a_{i}$.

3. [T, 3.4] If $a \wedge b$ exists, then $a \vee b$ exists and $(a \wedge b)+(a \vee b)=a+b$.

4. [T, 3.23] If $n \leq \infty$ and $a_{i} \wedge a_{j}=0$, for all $i \neq j<n$, then $\bigvee_{i<n} a_{i}=$ $\sum_{i<n} a_{i}$.

5. [T, 3.16,3.17] For any $n \leq \infty, a=\bigvee_{i<n} a_{i}$ iff for each $i, a_{i} \leq a$, and if $b$ is such that for every $i, a_{i} \leq b \leq a$, then $a=b$. Similarly for $\bigwedge_{i<n} a_{i}$.

6. $[\mathrm{T}, 4.3]$ For any $a$, we have either $a=2 a=3 a=\cdots=\infty a$ or $a<2 a<3 a<\cdots<\infty a$. 
7. [T, 2.28] If $S, T \subseteq A$ are nonempty countable and $\forall a \in S \forall b \in T(a \leq b)$, then there is c such that $\forall a \in S \forall b \in T(a \leq c \leq b)$. [T, 2.30] Moreover, if $S, T \subseteq A$ are nonempty countable and $\forall a \in S \forall b \in T(a \leq b)$ and $\forall a \in S \forall b \in T(a+d \leq e \leq b+d)$, then there is c with $e=c+d$ such that $\forall a \in S \forall b \in T(a \leq c \leq b)$

8. [T, 2.35] If $m \neq 0, n$ are finite and $m a+n c \leq m b+n c$, then $a+c \leq b+c$ and similarly replacing $\leq b y=$. In particular, $m a \leq m b \Longrightarrow a \leq b$ and $m a=m b \Longrightarrow a=b$.

9. [T, 2.37] If $m, n \geq 1$ are finite and relatively prime, then $m a=n b \Longrightarrow$ $\exists c(a=n c \& b=m c)$.

10. [T, 1.37, 1.46, 1.47] We say that $b$ absorbs $a$ iff $a+b=b$. Then $\infty a$ is the smallest element that absorbs $a$. If $0<n \leq \infty$, then $b$ absorbs a iff $b$ absorbs na. If $n \leq \infty$, then $b$ absorbs $\sum_{i<n} a_{i}$ iff $\forall i<n\left(b\right.$ absorbs $\left.a_{i}\right)$.

11. [T, 2.16, 2.17] If $n \leq \infty$ and $a_{i}+c \leq b_{i}+c$, then $\sum_{i<n} a_{i}+c \leq \sum_{i<n} b_{i}+c$ and similarly replacing $\leq$ by $=$.

12. $[\mathrm{T}, 2.15] a+c=b+c \& c \leq a, b \Longrightarrow a=b$.

Some additional properties are established in $[\mathrm{Tr}]$.

Theorem 2.3 (Truss). The following hold in any cardinal algebra $\left\langle A,+, \sum\right\rangle$ :

1. [Tr, Theorem 3] Given $a_{1}, a_{2}, \ldots, a_{m}, m<\infty$, there is $n<\infty$ and a map $\varphi$ from $\left\{a_{1}, a_{2}, \ldots, a_{m}\right\}$ to the power set of $\{1,2, \ldots, n\}$ and elements $b_{1}, b_{2}, \ldots, b_{n}$ such that $a_{i} \leq a_{j} \Longleftrightarrow \varphi\left(a_{i}\right) \subseteq \varphi\left(a_{j}\right)$ and $a_{i}=\sum_{k \in \varphi\left(a_{i}\right)} b_{k}$.

2. [Tr, page 582] If $a \vee b, a \vee c, b \wedge c$ exist, then $a \vee(b \wedge c)=(a \vee b) \wedge(a \vee c)$ and similarly switching $\vee$ and $\wedge$.

(C) A subalgebra of a cardinal algebra $\left\langle A,+, \sum\right\rangle$ consists of a subset $B \subseteq A$ such that $B$ contains an element absorbed by all elements of $B, B$ is closed under,$+ \sum$, and $\left\langle B,+, \sum\right\rangle$ is a cardinal algebra (where,$+ \sum$ here are these operations restricted to $B$ ). For example, this is the case if $B$ contains 0 , is closed under,$+ \sum$, and is downwards closed under $\leq$. 
We note that the following sets form subalgebras in any cardinal algebra $\left\langle A,+, \sum\right\rangle$. Below an element $a \in A$ is called idempotent if $2 a=a$.

(i) For any idempotents $a, b$, the interval $I(a, b)=\{c: a \leq c \leq b\}$ and the "infinite" interval $I(a)=\{c: a \leq c\}$.

To see this, notice that if $a \leq x$, then $x$ absorbs $a$. So if in axiom (C) for cardinal algebras, we have $a^{\prime}+b^{\prime}=\sum_{n<\infty} c_{n}^{\prime}$, where $a \leq a^{\prime}, b^{\prime}, c_{n}^{\prime}$, and $\left(a_{n}^{\prime}\right),\left(b_{n}^{\prime}\right)$ are such that

$$
a^{\prime}=\sum_{n<\infty} a_{n}^{\prime}, b^{\prime}=\sum_{n<\infty} b_{n}^{\prime}, c_{n}^{\prime}=a_{n}^{\prime}+b_{n}^{\prime}
$$

then we can replace $a_{n}^{\prime}, b_{n}^{\prime}$ by $a_{n}^{\prime}+a, b_{n}^{\prime}+a$ without affecting these equalities. Similarly in axiom (D), we can replace $c$ by $c+a$.

(ii) The set $I d=\{\infty a: a \in A\}$ of all idempotents of $A$.

Again notice that if in axiom $(\mathrm{C}), a, b, c_{n}$ are idempotents, then we can replace $a_{n}, b_{n}$ by $\infty a_{n}, \infty b_{n}$. Also in axiom (D), if $a_{n}, b_{n}$ are idempotents, we can replace $c$ by $\infty c$.

It is easy to check that if $a$ is idempotent, then $a \vee b=a+b$. Indeed if $a \leq c, b \leq c$, then $c=a+a^{\prime}$, for some $a^{\prime}$. Then $c=a+a^{\prime}=a+a+a^{\prime}=a+c$, so $a+b \leq a+c=c$. Thus $\langle I d, \leq\rangle$ is an upper semilattice.

(D) If $\boldsymbol{S}=\langle S,+\rangle$ is an abelian semigroup with identity, a finitely additive measure (fam) on $\boldsymbol{S}$ is a homomorphism from $\boldsymbol{S}$ into the semigroup $\boldsymbol{R}=\langle[0, \infty],+\rangle$ (where $a+\infty=\infty+\infty=\infty$ ). We define again the partial pre-order $a \leq b \Longleftrightarrow \exists c(b=a+c)$ (this maybe not be a partial order). Also let for $n \in \mathbb{N}, a \in S, n a=a+a+\cdots+a$ ( $n$ times). The following is a well known result of Tarski (see, e.g., [W, Theorem 9.1]).

Theorem 2.4 (Tarski). Let $\boldsymbol{S}=\langle S,+\rangle$ be an abelian semigroup with identity and $a \in S$. Then the following are equivalent:

(i) $\forall n \in \mathbb{N}((n+1) a \not \leq n a)$,

(ii) There is a fam $\varphi$ such that $\varphi(a)=1$.

In the particular case of a cardinal algebra $\left\langle A,+, \sum\right\rangle$, for the semigroup $\langle A,+\rangle$ the condition (i) in Theorem 2.4 is equivalent to:

$\left(i^{*}\right)$ a is not idempotent, and thus we have the following corollary:

Corollary 2.5. Let $\left\langle A,+, \sum\right\rangle$ be a cardinal algebra and $a \in A$. Then the following are equivalent: 
(i) a is not idempotent,

(ii) There is a fam $\varphi$ on $\langle A,+\rangle$ such that such that $\varphi(a)=1$.

We also note the following result of Bhaskara Rao and Shortt [BRS]:

Theorem 2.6 (Bhaskara Rao-Shortt). If $\left\langle A,+, \sum\right\rangle$ is a cardinal algebra, then for each $a \neq b$, there is a fam $\varphi$ such that $\varphi(a) \neq \varphi(b)$.

\section{Cardinal algebras in the theory of Borel equivalence relations}

(A) Bireducibility types. In the sequel it will be convenient to admit the empty equivalence relation (on the empty space), denoted by $\emptyset$, as a Borel equivalence relation.

Definition 3.1. Let $\mathcal{E}$ be a class of Borel equivalence relations. We denote by $[\mathcal{E}]$ the quotient space of $\mathcal{E}$ by $\sim_{B}$, i.e., $[\mathcal{E}]=\{[E]: E \in \mathcal{E}\}$, where $[E]=$ $\left\{F \in \mathcal{E}: E \sim_{B} F\right\}$. We call $[E]$ the bireducibility type of $E$.

Given Borel equivalence relations $E, F$ on standard Borel spaces $X, Y$, resp., we let $E \sqsubseteq_{B}^{i} F$ mean that there is a Borel $F$-invariant set $B \subseteq Y$ so that $E \cong_{B} F$. Then $E \sqsubseteq_{B}^{i} F \& F \sqsubseteq_{B}^{i} E \Longrightarrow E \cong_{B} F$.

Finally, if $E_{i}, i<n$, where $n \leq \infty$, are Borel equivalence relations, with $E_{i}$ living on $X_{i}$, then we let $\bigoplus_{i<n} E_{i}$ be the equivalence relation on $\bigsqcup_{i<n} X_{i}=$ $\bigcup_{i<n} X_{i} \times\{i\}$ given by $(x, j) \bigoplus_{i<n} E_{i}(y, k) \Longleftrightarrow j=k \& x E_{j} y$. In particular, $E \oplus \emptyset \cong_{B} E$, for any Borel equivalence relation $E$.

Definition 3.2. Let $\mathcal{E}$ be a class of Borel equivalence relations such that:

1. $\emptyset \in \mathcal{E}$.

2. If $F \in \mathcal{E}$ and $E \sqsubseteq_{B}^{i} F$, then $E \in \mathcal{E}$. Equivalently, $\mathcal{E}$ is closed under $\cong_{B}$, and if $F \in \mathcal{E}$ lives on $Y$ and $X \subseteq Y$ is Borel $F$-invariant, then $F \mid X \in \mathcal{E}$.

3. If $F_{0}, F_{1}, F_{2}, \cdots \in \mathcal{E}$, then $\bigoplus_{n} F_{n} \in \mathcal{E}$.

4. IF $E, F_{0}, F_{1}, F_{2}, \cdots \in \mathcal{E}$ and $E \sim_{B} \bigoplus_{n} F_{n}$, then there are $E_{n} \in \mathcal{E}$ with $F_{n} \sim_{B} E_{n}$ such that $E \cong_{B} \bigoplus_{n} E_{n}$. Equivalently, if $E$ lives on $X$, then there is a Borel partition $X=\bigsqcup_{n} X_{n}$, with $X_{n}$ E-invariant, Borel such that $E \mid X_{n} \cong{ }_{B} F_{n}$.) 
5. If $E, F \in \mathcal{E}$ live on $X, Y$, resp., and $f: X \rightarrow Y$ is a Borel reduction of $E$ to $F$, then the F-saturation $B=[f(X)]_{F}$ of $f(X)$ is a Borel subset of $Y$ and $E \sim_{B} F \mid B$.

Then we say that $\mathcal{E}$ is a Tarskian class of Borel equivalence relations.

For any class $\mathcal{E}$ of Borel equivalence relations closed under $\bigoplus_{i<n}$, for $n \leq \infty$, we can define on $[\mathcal{E}]$ :

$$
\begin{gathered}
{[E]+[F]=[E \oplus F],} \\
\sum_{n}\left[E_{n}\right]=\left[\bigoplus_{n} E_{n}\right] .
\end{gathered}
$$

It is easy to check that these are well-defined.

Proposition 3.3. If $\mathcal{E}$ is a Tarskian class of Borel equivalence relations, then $\left\langle[\mathcal{E}],+, \sum\right\rangle$ is a cardinal algebra. Moreover, for $E, F \in \mathcal{E}, E \leq_{B} F \Longleftrightarrow$ $[E] \leq[F]$.

Proof. Axioms (A), (B) of Section 2, (A) are trivial to verify. We next show that axiom (C) holds. Let $E, F, G_{n} \in \mathcal{E}$ be such that $[E]+[F]=\sum_{n}\left[G_{n}\right]$, i.e, $[E \oplus F]=\sum_{n}\left[G_{n}\right]$ or $E \oplus F \sim_{B} \bigoplus_{n} G_{n}$. By property 4. in Definition 3.2, if $E$ lives on $X, F$ lives on $Y$, so that $E \oplus F$ lives on $X \sqcup Y$, we have $X \sqcup Y=\bigsqcup_{n} Z_{n}$, where $Z_{n}$ is Borel $(E \oplus F)$-invariant, and $(E \oplus F) \mid Z_{n} \sim_{B} G_{n}$. Let $E_{n}=(E \oplus F)\left|\left(Z_{n} \cap X\right), F_{n}=(E \oplus F)\right|\left(Z_{n} \cap Y\right)$. Then $E_{n}, F_{n} \in \mathcal{E}$, $\left[E_{n}\right]+\left[F_{n}\right]=\left[G_{n}\right]$ and $[E]=\sum_{n}\left[E_{n}\right],[F]=\sum_{n}\left[F_{n}\right]$.

Finally, we verify axiom (D). Let $F_{n}, G_{n} \in \mathcal{E}$ be such that $\left[F_{n}\right]=\left[G_{n}\right]+$ $\left[F_{n+1}\right]$. Consider $F_{0}$, which lives on $X_{0}$. Since $\left[F_{0}\right]=\left[G_{0}\right]+\left[F_{1}\right]=\left[G_{0} \oplus F_{1}\right]$, by property 4 . again, we have $X_{0}=Y_{0} \sqcup X_{1}$, where $Y_{0}, X_{1}$ are $F_{0}$-invariant, $F_{0} \mid Y_{0} \sim_{B} G_{0}$ and $F_{0} \mid X_{1} \sim_{B} F_{1}$. Since $\left[F_{1}\right]=\left[G_{1} \oplus F_{2}\right]$, we have $X_{1}=Y_{1} \sqcup X_{2}$, where $Y_{1}, X_{2}$ are $F_{1}$-invariant, thus $F_{0}$-invariant, $F_{1}\left|Y_{1}=F_{0}\right| Y_{1} \sim_{B} G_{1}$ and $F_{1}\left|X_{2}=F_{0}\right| X_{2} \sim_{B} F_{1}$, etc. Proceeding this way, we can find pairwise disjoint $F_{0}$-invariant sets $Y_{0}, Y_{1}, Y_{2}, \cdots \subseteq X_{0}$, so that if $X_{1}=X_{0} \backslash Y_{0}, X_{2}=X \backslash\left(Y_{0} \cup\right.$ $\left.Y_{1}\right), \ldots$, then $F_{0} \mid Y_{n} \sim_{B} G_{n}$ and $F_{0} \mid X_{n} \sim_{B} F_{n}$. Let $Y=X_{0} \backslash \bigcup_{n} Y_{n}$. Then $G=F_{0} \mid Y \in \mathcal{E}$ and for each $n,\left[F_{n}\right]=\left[F_{0} \mid X_{n}\right]=\left[F_{0} \mid Y\right]+\left[\bigoplus_{i<\infty} F_{0} \mid Y_{n+i}\right]=$ $[G]+\sum_{i<\infty}\left[G_{n+i}\right]$.

That $E \leq_{B} F \Longleftrightarrow[E] \leq[F]$ is obvious from property 5. in Definition 3.2 . 
Remark 3.4. Note that in verifying in Proposition 3.3 that $\left\langle[\mathcal{E}],+, \sum\right\rangle$ is a cardinal algebra, we only used properties 1-4. of Definition 3.2. Property 5. is only used to verify the last statement in that proposition.

(B) Tarskian classes of Borel equivalence relations. We next verify that various classes of Borel equivalence delations are Tarskian.

The following concept was introduced in a stronger form (requiring a ccc condition) in [K1]:

Definition 3.5. Let $E$ be a Borel equivalence relation on $X$. Then $E$ is idealistic if there is a map $C \in X / E \mapsto I_{C}$, assigning to each E-class $C$ a $\sigma$-ideal $I_{C}$ of subsets of $C$, with $C \notin I_{C}$, such that $C \mapsto I_{C}$ is Borel in the following sense: For each Borel set $A \subseteq X^{2}$, the set $A_{I} \subseteq X$ defined by $x \in A_{I} \Longleftrightarrow\left\{y \in[x]_{E}:(x, y) \in A\right\} \in I_{[x]_{E}}$ is Borel.

A typical example of an idealistic $E$ is a Borel equivalence relation induced by a Borel action of a Polish group.

By convention, we consider the empty equivalence relation to be idealistic. We now have the following result:

Theorem 3.6. The class $\mathcal{I}$ of idealistic Borel equivalence relations is Tarskian, so $\left\langle[\mathcal{I}],+, \sum\right\rangle$ is a cardinal algebra.

Proof. It is clear that $\mathcal{I}$ satisfies conditions 1., 2., 3. of Definition 3.2. We next verify condition 5 .

Lemma 3.7. Let $E \in \mathcal{I}$ live on nonempty $X$, let $F$ be a Borel equivalence relation living on $Y$ and let $f: X \rightarrow Y$ be a Borel reduction of $E$ to $F$. Then the $F$-saturation $B=[f(X)]_{F}$ of $f(X)$ is a Borel subset of $Y$ and $E \sim_{B} F \mid B$.

Proof. We will apply the "large section" uniformization theorem, see [K2, 18.6], in the form presented as Theorem $18.6^{*}$ in page 2 of:

http://math.caltech.edu/ kechris/papers/CDST-corrections.pdf

Define $P \subseteq Y \times X$ by $(y, x) \in P \Longleftrightarrow f(x) F y$. Then if $B=\operatorname{proj}_{Y}(P)$, clearly $B=[f(X)]_{F}$. Let $C \mapsto I_{C}$ witness that $E$ is idealistic and for each $y \in B$, let $I_{y}=I_{C}$, where $C=f^{-1}\left([y]_{F}\right)$. Clearly for any $y \in B$, $P_{y}=\{x:(y, x) \in P\}=C \notin I_{y}$, so condition (ii) in Theorem 18.6* is satisfied.

We next verify condition (i) in Theorem 18.6*. Let $R \subseteq Y \times X$ be Borel and define $Q \subseteq X^{2}$ by $\left(x, x^{\prime}\right) \in Q \Longleftrightarrow x E x^{\prime} \&\left(f(x), x^{\prime}\right) \in R$. Then $Q$ 
is Borel and for $y \in B$, we have $R_{y} \in I_{y} \Longleftrightarrow \exists x\left[f(x) F y \& x \in Q_{I}\right] \Longleftrightarrow$ $\forall x\left[f(x) F y \Longrightarrow x \in Q_{I}\right]$. Thus for $y \in B$, the condition $R_{y} \in I_{y}$ is both $\Sigma_{\mathbf{1}}^{\mathbf{1}}$ and $\Pi_{1}^{1}$, which verifies condition (i).

It follows that $B$ is Borel and there is Borel uniformization of $P$, which clearly gives a Borel reduction of $F \mid B$ to $E$, thus $E \sim_{B} F \mid B$.

It is clear that the proof of Lemma 3.7 also shows the following:

Lemma 3.8. Let $E \in \mathcal{I}$ live on nonempty $X$, let $F$ be a Borel equivalence relation living on $Y$ and let $f: X \rightarrow Y$ be a Borel reduction of $E$ to $F$. Then for any E-invariant Borel set $A \subseteq X$, the set $B=[f(A)]_{F}$ is Borel and there is a function $g: B \rightarrow A$ which is a Borel reduction of $F \mid B$ to $E \mid A$ and if $[f]: X / A \rightarrow B / F,[g]: B / F \rightarrow A / E$ are the induced functions, then $[g]=[f]^{-1}$.

Lemma 3.9. Let $E, F \in \mathcal{I}$ live on nonempty $X, Y$ and let $f: X \rightarrow Y$ be a Borel reduction of $E$ to $F$ and $g: Y \rightarrow X$ a Borel reduction of $F$ to $E$. Then there is an E-invariant Borel set $A \subseteq X$ such that if $B=[f(A)]_{F}$, then $B$ is Borel, $[f]: A / E \rightarrow B / F$ is (clearly) a bijection and $[g]:(Y \backslash B) / F \rightarrow$ $(X \backslash A) / E$ is also a bijection.

Proof. We follow the standard proof of the Schröder-Bernstein Theorem (see, e.g., [K2, Theorem 15.7]).

A subset $X^{\prime} \subseteq X / E$ will be called "Borel" if $\left\{x \in X:[x]_{E} \in X^{\prime}\right\}$ is Borel and similarly for $Y^{\prime} \subseteq Y / F$. Then, by Lemma 3.8, if $X^{\prime}$ is "Borel", then $[f]\left(X^{\prime}\right)$ is "Borel". Similarly if $Y^{\prime}$ is "Borel", so is $[g]\left(Y^{\prime}\right)$.

Define inductively $X_{n}^{\prime} \subseteq X / E, Y_{n}^{\prime} \subseteq Y / F$ as follows: $X_{0}^{\prime}=X / E, Y_{0}^{\prime}=$ $Y / F, X_{n+1}^{\prime}=[g][f]\left(X_{n}^{\prime}\right), Y_{n+1}^{\prime}=[f][g]\left(Y_{n}^{\prime}\right)$. Let also $X_{\infty}^{\prime}=\bigcap X_{n}^{\prime}, Y_{\infty}^{\prime}=\bigcap Y_{n}^{\prime}$ and put $A^{\prime}=X_{\infty}^{\prime} \cup \bigcup_{n}\left(X_{n}^{\prime} \backslash[g]\left(Y_{n}^{\prime}\right)\right)$ and $B^{\prime}=Y_{\infty}^{\prime} \cup \bigcup_{n}\left([f]\left(X_{n}^{\prime}\right) \backslash Y_{n+1}^{\prime}\right)$. Then $[f]\left(A^{\prime}\right)=B^{\prime}$ and $[g]\left((Y / F) \backslash B^{\prime}\right)=(X / E) \backslash A^{\prime}$. Put $A=\left\{x \in X:[x]_{E} \in\right.$ $\left.A^{\prime}\right\}, B=\left\{y \in Y:[y]_{F} \in B^{\prime}\right\}$.

We finally use these lemmas to verify condition 4 . of Definition 3.2. Let $E, F_{0}, F_{1}, F_{2}, \cdots \in \mathcal{I}$ and $E \sim_{B} \bigoplus_{n} F_{n}$. Say $E$ lives on $X$. Then we can find $Y$, a Borel partition $Y=\bigsqcup_{n} Y_{n}$ and $F$ a Borel equivalence relation on $Y$ such that $Y_{n}$ is $F$-invariant, $F \mid Y_{n} \sim_{B} F_{n}$ and $E \sim_{B} F$. Let $f: X \rightarrow Y$ and $g: Y \rightarrow X$ witness that $E \sim_{B} F$. By Lemma 3.9, there is an $E$ invariant Borel set $A \subseteq X$ such that if $B=[f(A)]_{F}$, then $B$ is Borel, $[f]: E / A \rightarrow B / F$ is a bijection and $[g]:(Y \backslash B) / F \rightarrow(X \backslash A) / E$ is also 
a bijection. Let $X_{n}=f^{-1}\left(B \cap Y_{n}\right) \cup\left[g\left((Y \backslash B) \cap Y_{n}\right)\right]_{E}$. Then by Lemma 3.8, $E\left|X_{n} \sim_{B} F\right| Y_{n} \sim_{B} F_{n}$ and clearly $X=\bigsqcup_{n} X_{n}$.

We next discuss various cardinal subalgebras of $\left\langle[\mathcal{I}],+, \sum\right\rangle$.

Theorem 3.10. (i) Let $\mathcal{C}$ be the class of countable Borel equivalence relations. Then $\left\langle[\mathcal{C}],+, \sum\right\rangle$ is a cardinal algebra.

(ii) Let $\mathcal{N S C}$ be the class of non-smooth countable Borel equivalence relations. Then $\left\langle[\mathcal{N S C}],+, \sum\right\rangle$ is a cardinal algebra.

(iii) Let $\mathcal{T}$ be the class of treeable countable Borel equivalence relations. Then $\left\langle[\mathcal{T}],+, \sum\right\rangle$ is a cardinal algebra. Similarly for the class $\mathcal{N S T}$ of nonsmooth treeable countable Borel equivalence relations.

(iv) Let $\mathcal{N U}$ be the class of non-universal countable Borel equivalence relations. Then $\left\langle[\mathcal{N U}],+, \sum\right\rangle$ is a cardinal algebra.

(v) Let $\mathcal{I} d \mathcal{C}$ be the class of idempotent countable Borel equivalence relations, i.e., those satisfying $E \oplus E \sim_{B} E$. Then $\left\langle[\mathcal{I} d \mathcal{C}],+, \sum\right\rangle$ is a cardinal algebra.

Proof. The proofs of (i) - (iii), (v) are straightforward, using Section 2, (C), the fact that the equivalence relation $E_{0}$ on $2^{\mathbb{N}}$ defined by $\left(x_{n}\right) E_{0}\left(y_{n}\right) \Longleftrightarrow$ $\exists n \forall m \geq n\left(x_{m}=y_{m}\right)$ is $\leq_{B}$-least among non-smooth Borel equivalence relations and the fact that there is a universal treeable countable Borel equivalence relation. For iv), we only need that the sum of a sequence of nonuniversal relations is non-universal, which is a result of Marks, see [MSS, Theorem 3.8].

In particular all the laws mentioned in Theorem 2.2 apply to all these cardinal algebras, which in particular include all the results in Theorem 1.1 (except for the last part of (iii) that will be dealt with in (C) below).

Remark 3.11. Consider the class $\mathcal{T}^{*}$ of Borel equivalence relations which ares treeable and essentially countable. Then by Hjorth [H1] every such relation $E$ admits a Borel countable complete section $A$ and then by an argument similar to that in the proof of [JKL, Theorem 3.3 (i)] it follows that $E \mid A$ is a treeable countable Borel equivalence relation and of course $E \mid A \sim_{B} E$. Therefore $[\mathcal{T}]=\left[\mathcal{T}^{*}\right]$ and part (iii) of Theorem 3.10 holds as well for $\mathcal{T}^{*}$.

We will finish this subsection with some remarks and open questions concerning the structure of some of the cardinal algebras of bireducibility types discussed here. 
Consider the cardinal algebra $\left\langle[\mathcal{N} \mathcal{S C}],+, \sum\right\rangle$. Its identity element is $\left[E_{0}\right]$, which is of course its $\leq$-least element. It also has a $\leq$-largest element, namely $\left[E_{\infty}\right]$, where $E_{\infty}$ is a universal countable Borel equivalence relation. It is known (see $[\mathrm{AK}]$ ) that $\langle[\mathcal{N S C}], \leq\rangle$ is very complicated, e.g., one can embed in it every Borel poset. However the following is open:

Problem 3.12. Is $\langle[\mathcal{N S C}], \leq\rangle$ a lattice? Equivalently (by Theorem 2.2, 3.) is it true that for any $a, b \in[\mathcal{N S C}], a \wedge b$ exists?

In an earlier version of this paper, we mentioned that in fact it seemed to be unknown whether there are any $\leq$-incomparable $a, b$ for which $a \wedge b$ exists. Such examples have been now found in [CK].

Consider next the cardinal algebra $\left\langle[\mathcal{N} \mathcal{U}],+, \sum\right\rangle$. We have here the following open problem:

Problem 3.13. Does $\langle[\mathcal{N U}], \leq\rangle$ have a $\leq$-largest element? If not, what is the shortest length of an unbounded wellordered subset of $\langle[\mathcal{N U}], \leq\rangle$ (it is clearly at least $\left.\aleph_{1}\right)$ ?

We have seen that $\langle[\mathcal{I} d \mathcal{C}], \leq\rangle$ is an upper semilattice (see Section 2, (C)).

Problem 3.14. Is $\langle[\mathcal{I} d \mathcal{C}], \leq\rangle$ a lattice?

If this is the case, then by Theorem 2.3, 2., it would be distributive. Note that by $[\mathrm{AK}]$ again, $\langle[\mathcal{I} d \mathcal{C}], \leq\rangle$ also embeds any Borel poset.

It is known that there are non-idempotent elements in $[\mathcal{N S C}]$, see $\mathrm{S}$. Thomas [Th]. In fact [Th, Lemma 3.4] gives a countable Borel equivalence relation $E \in \mathcal{N S C}$, which is not divisible by any $n>1$, i.e., there is no $F \in \mathcal{C}$ with $n F \sim_{B} F$. It follows, using Corollary 2.5, that there is a fam on $\langle[\mathcal{N S C}],+\rangle$ for which $\varphi([E])=1$, so $\varphi$ takes a finite value. Of course no such $\varphi$ can exist on $\langle[\mathcal{I} d \mathcal{C}],+\rangle$.

Finally, let $\mathcal{B}$ be the class of all Borel equivalence relations.

Problem 3.15. Is $\left\langle[\mathcal{B}],+, \sum\right\rangle$ a cardinal algebra?

As opposed to the last statement in Proposition 3.3 however, it is not the case that for Borel equivalence relations $E, F$ we have $E \leq_{B} F \Longleftrightarrow$ $[E] \leq[F]$. To see this we use the following result of Hjorth $[\mathrm{H}]$ : There is a Borel equivalence relation $E$ such that for some countable Borel equivalence relation $F$ we have $E \leq_{B} F$ but for no countable Borel equivalence relation $G$ we have $E \sim_{B} G$. We claim then that $[E] \not \subset[F]$. Otherwise there is a 
Borel equivalence relation $H$ such that $E \oplus H \sim_{B} F$. Let $E, H, F$ live of $X, Y, Z$, resp., so that $E \oplus H$ lives on $X \sqcup Y$. Let $F: Z \rightarrow X \sqcup Y$ witness that $F \leq_{B} E \oplus H$. Put $W=f^{-1}(X)$. Then $W$ is $F$-invariant and $F \mid W \sim_{B} E$, a contradiction.

(C) Borel isomorphism types. For each Borel equivalence relation $E \in \mathcal{B}$, denote by $[E]_{\cong}=\left\{F \in \mathcal{B}: E \cong{ }_{B} F\right\}$ its Borel isomorphism type. Let $[\mathcal{B}]_{\cong}=\{[E] \cong: E \in \mathcal{B}\}$ be the set of isomorphism types of Borel equivalence relations. We can define,$+ \sum$ on $[\mathcal{B}] \cong$ as before and then it is not hard to check that $\left\langle[\mathcal{B}]_{\cong},+, \sum\right\rangle$ is a cardinal algebra. It is also clear that in this cardinal algebra $[E] \cong \leq[F] \cong \Longleftrightarrow E \sqsubseteq_{B}^{i} F$. In particular all the laws in Theorem 2.2 hold in $\left\langle[\mathcal{B}]_{\cong},+, \sum\right\rangle$, including the cancellation law stated in Theorem 1.1, (iii).

\section{Cancellation fails for products}

We show here that the Cancellation Law

$$
n>1, n E \sim_{B} n F \Longrightarrow E \sim_{B} F,
$$

fails for products in the context of countable Borel equivalence relations. This answers a question of Andrew Marks, who raised it in connection with a discussion with Igor Pak on a related issue.

If $E, F$ are Borel equivalence relations, on $X, Y$, resp., their product $E \times F$ is the equivalence relation on $X \times Y$ defined by:

$$
(x, y) E \times F\left(x^{\prime}, y^{\prime}\right) \Longleftrightarrow x E x^{\prime} \& y F y^{\prime} .
$$

For $n \geq 1$ we let $E^{n}$ be the product of $n$ copies of $E$. We now have:

Theorem 4.1. There are countable Borel equivalence relations $E<_{B} F$ such that $E^{2} \sim_{B} F^{2}$.

The proof was inspired by the result of Tarski in cardinal arithmetic that states that the Axiom of Choice is equivalent to the statement: For any two infinite cardinals $\kappa, \lambda\left(\kappa^{2}=\lambda^{2} \Longrightarrow \kappa=\lambda\right)$; see [J, Theorem 11.8]. As opposed to the proof of Tarski's Theorem, that makes use of the Hartogs number of an infinite cardinal, the proof of Theorem 4.1 uses ideas of ergodic theory and geometric group theory.

The main idea for the construction of a pair $E, F$ as in Theorem 4.1 is based on the following result: 
Theorem 4.2. Suppose $R, S$ are Borel equivalence relations on standard Borel spaces $X, Y$, resp., such that:

(i) $X / R$ and $Y / S$ are infinite.

(ii) There are probability Borel measures $\mu, \nu$ on $X, Y$, resp., such that $R$ is $\mu$-ergodic, $S$ is $\nu$-ergodic and for any $R$-invariant Borel set $A$ with $\mu(A)=1$ and any $S$-invariant Borel set $B$ with $\nu(B)=1$, we have $R \mid A \not_{B} S$ and $S \mid B \not \mathbb{Z}_{B} R$.

(iii) $R^{2} \sim_{B} R, S^{2} \sim_{B} S$.

Let $E=R \oplus S$ and $F=R \times S$. Then $E<_{B} F$ but $E^{2} \sim_{B} F^{2}$.

Proof. First notice that $R \oplus S \leq_{B} R \times S$. Indeed $R \oplus S$ lives in the direct sum $X \sqcup Y$. Fix $\left(x_{0}, y_{0}\right) \in X \times Y$. Since clearly $R \leq_{B} R \mid\left(X \backslash\left[x_{0}\right]_{R}\right)$, and similarly for $S$, it is enough to show that $R\left|\left(X \backslash\left[x_{0}\right]_{R}\right) \oplus S\right|\left(Y \backslash\left[y_{0}\right]_{S}\right) \leq_{B}(R \times S)$. Let $Z=\left(X \backslash\left[x_{0}\right]_{R}\right) \sqcup\left(Y \backslash\left[y_{0}\right]_{S}\right)$. Then define $f: Z \rightarrow X \times Y$ by $f(x)=$ $\left(x, y_{0}\right), f(y)=\left(x_{0}, y\right)$. Then $f$ is Borel reduction of $R\left|\left(X \backslash\left[x_{0}\right]_{R}\right) \oplus S\right|\left(Y \backslash\left[y_{0}\right]_{S}\right)$ to $R \times S$.

Clearly $(R \times S)^{2} \sim_{B}\left(R^{2} \times S^{2}\right) \sim_{B} R \times S$. Also $(R \oplus S)^{2} \sim_{B} R^{2} \oplus 2 R \times$ $S \oplus S^{2} \geq_{B} R \times S$. Also note that, denoting by 2 the equality relation on a set of cardinality 2 , we have $2 \times R=2 R$ and $2 \leq_{B} R$, so $2 R \leq_{B} R^{2} \sim_{B} R$, so $2 R \sim_{B} R$. Thus we have $(R \oplus S)^{2} \sim_{B} R \oplus S \oplus(R \times S) \leq_{B} 2 R \times S \sim_{B} R \times S$, so $(R \oplus S)^{2} \sim_{B}(R \times S)^{2}$.

It remains to show that $R \oplus S<_{B} R \times S$. Otherwise, assume that $R \times S \leq_{B} R \oplus S$, towards a contradiction, and let $f: X \times Y \rightarrow X \sqcup Y$ witness that. Put $X_{0}=f^{-1}(X), Y_{0}=f^{-1}(Y)$, so that $X_{0} \sqcup Y_{0}=X \times Y$. Also $X_{0}, Y_{0}$ are $(R \times S)$-invariant and $(R \times S)\left|X_{0} \leq_{B} R,(R \times S)\right| Y_{0} \leq_{B} S$.

Claim. $R \times S$ is $(\mu \times \nu)$-ergodic.

Proof of claim. Let $A \subseteq X \times Y$ be $(R \times S)$-invariant. Then for each $x$ the section $A_{x}$ is $S$-invariant and $x R x^{\prime} \Longrightarrow A_{x}=A_{x^{\prime}}$. Thus the function $x \rightarrow \nu\left(A_{x}\right) \in\{0,1\}$ is $R$-invariant, thus constant $\mu$-a.e. If this constant value is 1 , then by Fubini $\mu \times \nu(A)=1$, while if it is $0, \mu \times \nu(A)=0$.

So we have two possibilities: $\mu \times \nu\left(X_{0}\right)=1$ or $\mu \times \nu\left(X_{1}\right)=1$. In the first case, there is $x$ such that $\nu\left(\left(X_{0}\right)_{x}\right)=1$ and of course $\left(X_{0}\right)_{x}$ is $S$-invariant. The map $y \in\left(X_{0}\right)_{x} \mapsto f(x, y)$ witnesses that $S \mid\left(X_{0}\right)_{x} \leq_{B} R$, which is a contradiction. The second case is similar.

Thus to complete the proof of Theorem 4.1, it remains to construct examples of countable Borel equivalence relations $R, S$ satisfying conditions (i), (ii), (iii) of Theorem 4.2 . 
We will use the following result from group theory that was explained to us by Simon Thomas in response to a question by one of the authors.

Theorem 4.3 (Yu. A. Olshanskii). There is a countable, torsion free, simple group $\Gamma$ with property $(\mathrm{T})$ and an infinite countable, torsion, simple group $\Delta$ with property $(\mathrm{T})$.

Proof. For the convenience of the reader, we will give a sketch of the proof based on the results of Olshanskii [O].

Fix a countable group $G$ which is torsion free, hyperbolic and has property (T) (see,e.g., [DC, Proposition 2]). By [O, Corollary 1], there is a torsion free quotient $\Gamma$ of $G$ all of whose non-trivial proper subgroups are cyclic. Thus $\Gamma$ has property $(\mathrm{T})$ and we next check that it is simple. By the proof of Corollary 1 in [O, pages 403-404], the center of $\Gamma$ is trivial. If $N$ is a nontrivial proper normal subgroup, then it is not contained in the center, so by looking at the conjugation action of $\Gamma$ on $N$, we have a non-trivial homomorphism of $\Gamma$ into the automorphism group of $N$, which is a 2-element group, so $\Gamma$ has a subgroup of index two, a contradiction.

To define $\Delta$, use [O, Corollary 4] to find an infinite quotient $G_{1}$ of $G$ which is quasi-finite (i.e., every proper subgroup is finite). If $N \triangleleft G_{1}$ is a proper normal subgroup, by looking again at the conjugation action of $G_{1}$ on the finite group $N$, we conclude that $N \leq Z\left(G_{1}\right)$. Put $\Delta=G_{1} / Z\left(G_{1}\right)$. Then $\Delta$ is infinite, torsion, simple and has property $(\mathrm{T})$.

Fix the groups $\Gamma, \Delta$ as in Theorem 4.3. Put $\Gamma^{*}=\Gamma \oplus \Gamma \oplus \ldots, \Delta^{*}=\Delta \oplus$ $\Delta \oplus \ldots$ Then $\Gamma^{*} \times \Gamma^{*} \cong \Gamma^{*}$ and $\Delta^{*} \times \Delta^{*} \cong \Delta^{*}$. Since every homomorphism from $\Gamma$ to $\Delta$ is trivial and vice versa, it follows that every homomorphism of $\Gamma$ to $\Delta^{*}$ is trivial and vice versa.

For any countable group $G$, consider the shift action of $G$ on $[0,1]^{G}$ restricted to its free part and let $F_{G}$ be the corresponding equivalence relation. Put now $R=F_{\Gamma^{*}}$, which lives in $X$, and $S=F_{\Delta^{*}}$, which lives in $Y$. We will verify that these satisfy the conditions of Theorem 4.2 . Let $\mu$ be the product measure on $[0,1]^{\Gamma^{*}}$ restricted to $X$ and similarly define $\nu$ on $Y$.

Condition (i) of Theorem 4.2 is obvious. Also $R$ is $\mu$-ergodic and similarly $S$ is $\nu$-ergodic. We will next verify that if $A \subseteq X$ is $R$-invariant and has $\mu$ measure 1, then $R \mid A \not \leq_{B} S$ (and vice versa).

For that we will use the superrigidity result of Popa $[\mathrm{P}]$ (see also [K3, Theorem 30.5] for an exposition), which asserts that if $G$ is a countable infinite group with property (T), $H$ is a countable group, and $\alpha$ is a Borel 
cocycle of the shift action of $G$ on $[0,1]^{G}$ into $H$, then $\alpha$ is cohomologous to a homomorphism from $G$ to $H$.

So assume that $f$ is a Borel reduction of $R \mid A$ to $S$. Viewing $\Gamma$ in the obvious way as a subgroup of $\Gamma^{*}$, this gives the following Borel cocycle $\alpha(\gamma, x)$ from the restriction to $\Gamma$ of the shift action of $\Gamma^{*}$ on $[0,1]^{\Gamma^{*}}$ into $\Delta^{*}: f(\gamma \cdot x)=$ $\alpha(\gamma, x) \cdot f(x)$. Since this action of $\Gamma$ is isomorphic to the shift action of $\Gamma$ on $[0,1]^{\Gamma}$, by Popa's Theorem there is a Borel function $\pi: X \rightarrow \Gamma^{*}$ such that $\alpha(\gamma, x)=\pi(\gamma \cdot x) \pi(x)^{-1}$, $\mu$-a.e. Let $g(x)=\pi(x)^{-1} \cdot f(x)$. Then $g$ is also a reduction of $R \mid A$, $\mu$-a.e., to $S$ and $g(\gamma \cdot x)=g(x)$. By ergodicity $g$ must be constant $\mu$-a.e., a contradiction.

Finally we verify condition (iii) of Theorem 4.2. We will show that $R^{2} \sim_{B}$ $R$ and similarly for $S$.

We have that $R^{2}=\left(F_{\Gamma^{*}}\right)^{2}$, which is an equivalence relation on $X^{2}$ induced by the following free action of $\Gamma^{*} \times \Gamma^{*}$ on $X^{2}:(\delta, \epsilon) \cdot(x, y)=(\delta \cdot x, \epsilon \cdot y)$. Any free Borel action of a countable group $G$ on an uncountable standard Borel space $Z$, which we can assume it is the interval $[0,1]$, can be embedded in a Borel way to the shift action of $G$ on $[0,1]^{G}$ via: $z \mapsto\left(g \rightarrow g^{-1} \cdot z\right)$. Therefore $R^{2} \leq_{B} F_{\Gamma^{*} \times \Gamma^{*}} \cong_{B} F_{\Gamma^{*}}=R$, so $R \sim_{B} R^{2}$.

Remark 4.4. There is a Baire category analog of Theorem 4.2, where $X, Y$ are now Polish spaces and $R, S$ are generically ergodic and for any $R$-invariant Borel comeager set $A$ and any every $S$-invariant Borel comeager set $B$, we have $R \mid A \mathbb{Z}_{B} S$ and $S \mid B \mathbb{Z}_{B} R$. Using this one can show that for $R=E_{0}^{\mathbb{N}}, S=E_{1}$, if $E=R \oplus S, F=R \times S$, then $E<_{B} F$ but $E^{2} \sim_{B} F^{2}$. (Here $E_{1}$ is the equivalence relation on $\mathbb{R}^{\mathbb{N}}$ defined by $\left(x_{n}\right) E_{1}\left(y_{n}\right) \Longleftrightarrow \exists n \forall m \geq n\left(x_{m}=y_{m}\right)$ and $E_{0}^{\mathbb{N}}$ is the equivalence relation on $\left(2^{\mathbb{N}}\right)^{\mathbb{N}}$ defined by $\left(x_{n}\right) E_{0}^{\mathbb{N}}\left(y_{n}\right) \Longleftrightarrow \forall n\left(x_{n}=y_{n}\right)$.)

Remark 4.5. One can also consider the set $\mathcal{C}$ as in Theorem 3.10, 1., with the operation of multiplication: $[E] \cdot[F]=[E \times F]$. It forms an abelian group with identity (the equivalence relation on a singleton space). If $E_{\infty T}$ is the universal treeable countable Borel equivalence relation, then, by [HK, Theorem 8.1], we have $E_{\infty T}<_{B} E_{\infty T}^{2}<_{B} E_{\infty T}^{3}<_{B} \cdots$, so, by Theorem 2.4, there is a fam on $\langle\mathcal{C}, \cdot\rangle$ such that $\varphi\left(\left[E_{\infty T}\right]=1\right.$.

Acknowledgments. Research partially supported by NSF Grant DMS1464475. We would like to thank Simon Thomas for his help with Theorem 4.3 . 


\section{References}

[AK] S. Adams and A.S. Kechris, Linear algebraic groups and countable Borel equivalence relations, J. Amer. Math. Soc., 13 (2000), 104137.

[BRS] K.P.S. Bhaskara Rao and R.M. Shortt, Weak cardinal algebras, Papers on general topology and applications (Brookville, NY, 1990), Ann. New York Acad. Sci., 659, New York Acad. Sci., New York, 1992, $156-162$.

[CK] R. Chen and A.S. Kechris, Structurable equivalence relations, in preparation.

[DC] Y. de Cornulier, A note on quotients of word hyperbolic groups with property (T), arXiv:math/0504193v3.

[H] G. Hjorth, Bi-Borel reducibility of essentially countable Borel equivalence relations. J. Symbolic Logic, 70(3) (2005), 979-992.

[H1] G. Hjorth, Selection theorems and treeability, Proc. Amer. Math. Soc., 136(10) (2008), 3647-3653.

[HK] G. Hjorth and A.S. Kechris, Rigidity theorems for actions of product groups and countable Borel equivalence relations, Memoirs of the Amer. Math. Soc., Vol. 177, No. 833, 2005.

[JKL] S. Jackson, A.S. Kechris and A. Louveau, Countable Borel equivalence relations J. Math. Logic, 2 (2002), 1-80.

[J] T.J. Jech, The Axiom of Choice, North-Holland, 1973.

[K1] A.S. Kechris, Countable sections for locally compact group actions, Ergod. Th. \& Dynam. Syst., 12 (1992), 283-295.

[K2] A.S. Kechris, Classical Descriptive Set Theory, Springer-Verlag, 1995.

[K3] A.S. Kechris, Global aspects of Ergodic Group Actions, Amer. Math. Soc., 2010. 
[MSS] A. Marks, T. Slaman and J. Steel, Martin's conjecture, arithmetic equivalence, and countable Borel equivalence relations, Ordinal Definability and Recursion Theory: The Cabal Seminar, Volume III, Edited by A. S. Kechris, B. Löwe, J. R. Steel, Cambridge Univ. Press, to appear, 2015.

[O] Yu. A. Olshanskii, On residualing homomorphisms and $G$-subgroups of hyperbolic groups. Internat. J. Algebra Comput., 3(4) (1993), 365-409.

[P] S. Popa, Cocycle and orbit equivalence superrigidity for malleable actions of $w$-rigid groups, Invent. Math., 170(2) (2007), 243-295.

[T] A. Tarski, Cardinal Algebras, Oxford University Press, 1949.

[Th] S. Thomas, Some applications of superrigidity to Borel equivalence relations, DIMACS Ser. Discrete Math. Theoret. Comput. Sci., 58, Amer. Math. Soc., Providence, RI, 2002, 129-134,

[Tr] J. Truss, Convex sets of cardinals, Proc. London Math. Soc., 3(27) (1973), 577-599.

[W] S. Wagon, The Banach-Tarski Paradox, Cambridge University Press, 1993

Department of Mathematics

California Institute of Technology

Pasadena, CA 91125

kechris@caltech.edu

Mathematics Department

Occidental College (M-15)

1600 Campus Rd

Los Angeles, CA 90041-3314

hlm628@gmail.com 